\title{
Enhancement of the incretin pathway in response to bariatric surgery is important for restoration of beta cell function. Reply to Komatsu M, Aizawa T [letter]
}

\author{
S. Salinari • A. Bertuzzi • A. Iaconelli • M. Manco • \\ G. Mingrone
}

Received: 30 September 2008 / Accepted: 5 November 2008 / Published online: 5 December 2008

(C) Springer-Verlag 2008

Keywords Bariatric surgery - Beta cell secretion . Mathematical model $\cdot$ Type 2 diabetes mellitus

\author{
Abbreviations \\ BPD bariatric surgery \\ ISR insulin secretion rate
}

To the Editor: In response to the letter of Komatsu and Aizawa [1] concerning our recent paper in Diabetologia [2], we wish to make the following points.

Because of space constraints we were not able to clarify the roles of the different parameters included in our model in detail. Although, as noted by Komatsu and Aizawa, glucose and NEFA are the only inputs used to obtain the insulin secretion rate (ISR), the model response is also determined by the values of the parameters $p_{1}, p_{2}$ and $p_{3}$. These parameters

S. Salinari $(\bowtie)$

Department of Systems Analysis and Informatics,

University of Rome 'La Sapienza',

Via Ariosto 25,

00185 Rome, Italy

e-mail: salinari@dis.uniroma1.it

\section{A. Bertuzzi}

Institute of Systems Analysis and Computer Science, CNR, Rome, Italy

A. Iaconelli $\cdot$ G. Mingrone

Institute of Internal Medicine, Catholic University,

School of Medicine,

Rome, Italy

M. Manco

Liver Unit, Bambino Gesù Hospital and Research Institute,

Rome, Italy are estimated by fitting the model to the C-peptide experimental data. The equation for estimating ISR contains a function that includes both glucose and NEFA levels. This function contains the parameters $p_{2}$ and $p_{3}$, which are related to beta cell fatty acid oxidation. The parameter $p_{1}$ acts as a multiplier of this function, and represents a further factor involved in the potentiation of the ISR. Incretin action, in particular, is implicitly accounted for by the parameter $p_{1}$ of the model. The value of $p_{1}$ in a given experimental setting is thus related to factors other than NEFA and glucose that determine the ISR.

We have found that the parameters $p_{2}$ and $p_{3}$ markedly change after bariatric surgery (BPD), becoming very close to control values (Table 1). It therefore appears that defective $\beta$-oxidation is reversed after BPD. However, we do not imply that this is the major factor responsible for the improvement in ISR. In fact, after BPD, the NEFA level is unchanged whereas the glucose concentration is decreased, probably because of the increase in insulin sensitivity. Parameter $p_{1}$ is doubled after BPD, but does not reach the value found in controls. We interpret this fact as evidence that an incretin-dependent increase in beta cell sensitivity to glucose-NEFA has occurred. The incretin effect implicitly resides in the value of parameter $p_{1}$. Figure $3 \mathrm{a}$ of our paper [2] shows the effects of BPD on the model response.

In a previous article we applied this model to data from healthy volunteers during a hyperglycaemic clamp after either water or butter ingestion [3]. After the water load, the parameter $p_{1}$ was half the average value observed using the $24 \mathrm{~h}$ multiple-meal test. Butter alone was able to double parameter $p_{1}$, possibly via the incretin effect. In fact, in this paper we suggested that the potentiation of insulin secretion mediated by gastrointestinal hormones does not occur after water ingestion but can take place after a butter test meal [3]. 
We appreciate the suggestion made by Komatsu and Aizawa [1] regarding the effect of NEFA on the replenishment of the readily releasable pool of insulin granules during the second phase of insulin secretion. We recently proposed a mathematical model of insulin granule trafficking in the beta cell [4] in which the dynamics of the various granule pools, including reserve, docked and primed, immediately releasable and fused granules, are represented. Unfortunately, the paper by Aizawa and Komatsu [5] escaped our attention. Extension of the model to include the effect of NEFA would be of great interest.

Duality of interest The author declares that there is no duality of interest associated with this manuscript.

\section{References}

1. Komatsu M, Aizawa T (2009) Enhancement of the incretin pathway in response to bariatric surgery is important for restoration of beta cell function. Diabetologia doi:10.1007/s00125-008-1230-6

2. Salinari S, Bertuzzi A, Iaconelli A, Manco M, Mingrone G (2008) Twenty-four hour insulin secretion and beta cell NEFA oxidation in type 2 diabetic, morbidly obese patients before and after bariatric surgery. Diabetologia 51:1276-1284

3. Salinari S, Bertuzzi A, Manco M, Mingrone G (2007) NEFAglucose comodulation model of $\beta$-cell insulin secretion in 24-h multiple-meal test. Am J Physiol Endocrinol Metab 292: E1890-E1898

4. Bertuzzi A, Salinari S, Mingrone G (2007) Insulin granule trafficking in $\beta$-cells: mathematical model of glucose-induced insulin secretion. Am J Physiol Endocrinol Metab 293:E396E409

5. Aizawa T, Komatsu M (2005) Rab27a: a new facet in $\beta$-cell metabolism-secretion coupling. J Clin Invest 115:227-230 